\title{
Real-Time Dynamics during Recharging Cycles
}

C. A. Orme, J. Keist, S. G. Torres, B. El-dasher, J. Evans, P. Wright, F. M. Ross, D. Steingart

November 29, 2012

Real-Time Dynamics during Recharging Cycles Honolulu, HI, United States October 7, 2012 through October 12, 2012 
This document was prepared as an account of work sponsored by an agency of the United States government. Neither the United States government nor Lawrence Livermore National Security, LLC, nor any of their employees makes any warranty, expressed or implied, or assumes any legal liability or responsibility for the accuracy, completeness, or usefulness of any information, apparatus, product, or process disclosed, or represents that its use would not infringe privately owned rights. Reference herein to any specific commercial product, process, or service by trade name, trademark, manufacturer, or otherwise does not necessarily constitute or imply its endorsement, recommendation, or favoring by the United States government or Lawrence Livermore National Security, LLC. The views and opinions of authors expressed herein do not necessarily state or reflect those of the United States government or Lawrence Livermore National Security, LLC, and shall not be used for advertising or product endorsement purposes. 


\author{
Real-Time Dynamics during Recharging Cycles \\ J. Keist ${ }^{\mathrm{ab}}$, B. El Dasher ${ }^{\mathrm{a}}$, S. G. Torres ${ }^{\mathrm{a}}$, J. Evans ${ }^{\mathrm{b}}$, P. Wright ${ }^{\mathrm{b}}$, F. M. Ross ${ }^{\mathrm{c}}$, D. Steingart ${ }^{\mathrm{d}}$, \\ and C. A. Orme ${ }^{\mathrm{a}}$ \\ ${ }^{a}$ Lawrence Livermore National Lab, California, 94550, USA \\ ${ }^{\mathrm{b}}$ University of California, Berkeley, California 94720, USA \\ ${ }^{\mathrm{c}}$ IBM T. J. Watson Research Center, Yorktown Heights, New York 10598, USA \\ ${ }^{\mathrm{d}}$ City College New York, New York, New York 10031, USA
}

\begin{abstract}
This paper describes preliminary results from in situ electrochemical atomic force microscopy (EC AFM) and in situ electrochemical small angle x-ray scattering (EC USAXS) studies. Experiments are designed to quantify the morphological evolution during charge and discharge cycles. From the AFM data we measure feature shapes, nucleation density, island distributions and quantify surface roughness using height-height correlation functions. The USAXS scattering data is modeled as a distribution of islands. The goal of our project is to link early time nucleation events with the onset of large-scale instabilities such as dendrites.
\end{abstract}

\title{
Introduction
}

Current energy needs have triggered a resurgence in battery research, particularly to develop better rechargeable batteries for vehicles and for leveling intermittent energy loads. Batteries work by releasing energy (a flow of electrons) as metals are oxidized. Rechargeable batteries require a reversible process in which the oxide is converted back to a metal by flowing a current. In other words, rechargeable batteries operate by continuously cycling crystal growth and dissolution. This project images the anode interface during charging and discharging. We hypothesize that quantifying the morphological evolution will provide new mechanistic details of electrolyte-surface interactions. This work will address an important gap in our understanding of how variations in electrolyte chemistry impact the morphology and kinetics of electrified battery interfaces. The overarching goal of this study is to increase the lifetime of rechargeable batteries by preventing failures due to dendrite formation and other interface instabilities.

This paper describes our initial baseline experiments on zinc anodes in alkaline electrolytes that may be part of secondary zinc batteries or zinc flow batteries. This baseline will be used to compare with alkaline electrolytes containing additives and also to compare with other electrolytes such as ionic liquids. Experiments are designed to quantify the morphological evolution during charge and discharge cycles by coupling in situ electrochemical atomic force microscopy (EC AFM) studies with electrochemical small angle x-ray scattering (EC USAXS). 


\section{Methods}

This paper describes in situ electrochemical AFM and in situ electrochemical USAXS of zinc deposition on a copper substrate from a 9 Molar $\mathrm{KOH}$ solution (EM Industries) with $0.6 \mathrm{M} \mathrm{ZnO}$ (Mallinkrodt).

Zinc films were deposited using galvanostatic pulses using a Gamry Series G 300/750 Potentiostat/Galvanostat. Both the counter and reference electrodes were zinc wire. Between pulses the surface was held potentiostatically at $+0.1 \mathrm{~V}$ with respect to the zinc wire reference electrode to prevent either the copper substrate or the zinc islands from evolving. A sequence of pulses and holds were maintained by using a script. The purpose of these experiments was to survey a range of current densities that would allow the nanometer to micron scale to be investigated across instrument platforms. For this reason we chose a sequence of ten ten-second current pulses: three each at $-1,-2,-5$ $\mathrm{mA} / \mathrm{cm}^{2}$, followed by one pulse at $-10 \mathrm{~mA} / \mathrm{cm}^{2}$. The current was then reversed to strip the zinc back off of the surface. The same script was used for both AFM and USAXS experiments.

In situ AFM imaging was performed using an Asylum MFP3D microscope equipped with a PEEK electrochemical cell. AFM cantilevers (silicon nitride) and tips (either silicon or silicon nitride) are marginally stable in $9 \mathrm{M} \mathrm{KOH}$ but last for the duration of the experiment if contact is avoided when the surface is electrified. During current pulses the AFM head was lifted away from the surface to avoid electrical shielding and to maximize solution access to the region of interest. After the current pulse, the tip was repositioned within 0.1 micron of the original location. Imaging was performed using tapping mode to lessen tip-blunting.

In situ USAXS was performed at the Advance Photon Source on the ChemMatCARS sector on beamline 15-ID. The purpose built fluid cell ${ }^{1}$ accommodates a 12.5 micron thick copper foil that is placed perpendicular to the xray flux with approximately laminar fluid flow across both faces. Scattering occurs at both surfacesolution interfaces with a total area of approximately $15 \mathrm{~mm}^{2}$. Roughness stems from the intrinsic roughness of the substrate and the zinc islands that nucleate and grow as the experiment proceeds. The USAXS scattering geometry provides information about the in-plane feature dimensions. Data were analyzed using Irena Modeling II software (version 2.47). ${ }^{2}$ We used a power law fit (unified fit) to describe the low-q scatter intensity and a log-normal spheroid distribution to describe the high-q, Guinier region that develops as zinc is deposited.

\section{Preliminary Results and Discussion}

AFM images were obtained of the initial surface and after each current pulse. During imaging (between pulses) the surface was held at $+0.1 \mathrm{~V}$ with respect to the zinc wire reference electrode. This was necessary to prevent the copper from spontaneously transforming to copper oxide in the strong alkaline environment. At this holding potential, 
sequential AFM images showed that the surface morphology did not evolve over at least an hour. This allowed us to "quench" the surface dynamics while we captured AFM images and USAXS scans.

Figure 1 shows images after the first current pulse at 1,2,5, and $10 \mathrm{~mA} / \mathrm{cm}^{2}$. (This represents a subset of the images captured). All images are $2 \mu \mathrm{m}$ x $2 \mu \mathrm{m}$ with a zscale of $200 \mathrm{~nm}$.
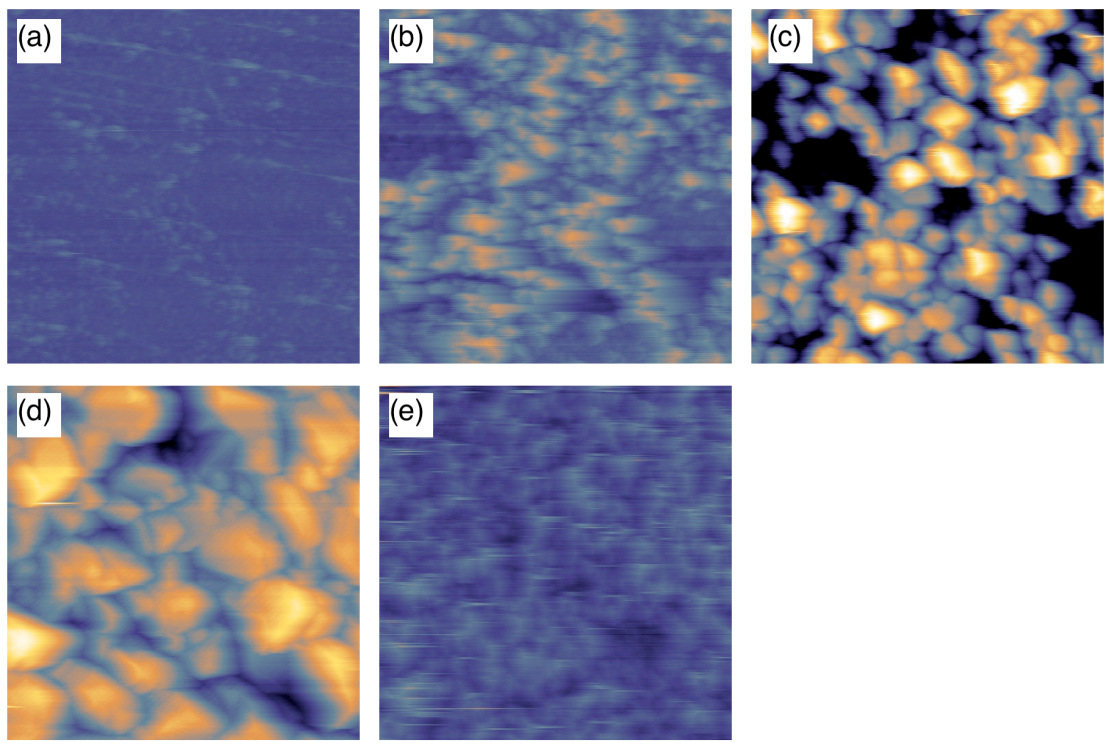

Figure 1. AFM images $(2 \mu \mathrm{m} \times 2 \mu \mathrm{m})$ of the same area after a sequence of voltage pulses. a) $-1 \mathrm{~mA} / \mathrm{cm}^{2}$, b) $-2 \mathrm{~mA} / \mathrm{cm}^{2}$, c) $-5 \mathrm{~mA} / \mathrm{cm}^{2}$, d) $-10 \mathrm{~mA} / \mathrm{cm}^{2}$, and e) after stripping zinc islands by reversing current.

The images show zinc islands nucleating, growing and finally coalescing as deposition proceeds. Nucleation occurs both on surface steps as well as surface terraces with islands that have an approximately 0.2 aspect ratio (wider than they are tall). The root mean square roughness (Figure 2a) increases as the deposition proceeds but dips between the first and second $5 \mathrm{~mA} / \mathrm{cm}^{2}$ pulse, indicating that this is where the islands begin to coalesce.

The autocorrelation functions (Figure 2b), which provide information on lateral feature sizes, demonstrate that the lateral dimensions grow and then eventually saturate as more zinc is deposited. At saturation, the average lateral feature size is approximately $200 \mathrm{~nm}$ (as estimated from the zero-crossing point). It is the lateral feature size that should be compared to the USAXS scattering data.

We note that the morphology was more difficult to image for the surfaces obtained with -1 and $-2 \mathrm{~mA} / \mathrm{cm}^{2}$ pulses. This may be due to a different chemistry that creates a more "sticky" tip-surface interaction. We investigated the surface chemistry using in situ Raman (data not shown) by comparing spectra for surfaces grown with $-1,-2$, and $-10 \mathrm{~mA} / \mathrm{cm}^{2}$ but did not find evidence of oxides or hydroxides that might cause differences in tip-surface interactions. Future work will quantify this effect using force spectroscopy. 
(a)

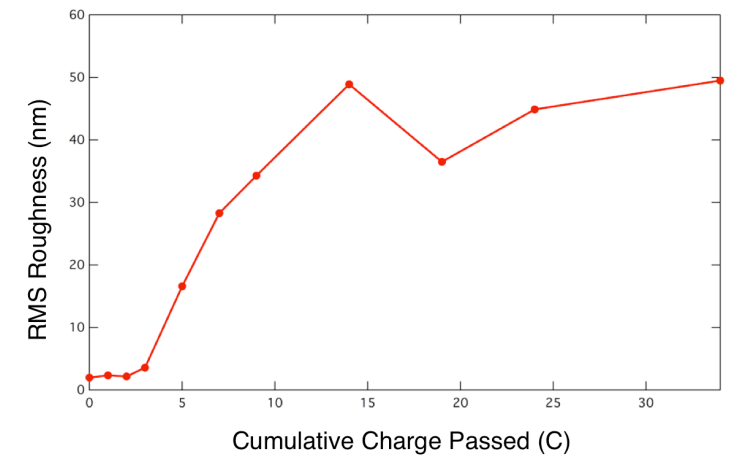

(b)

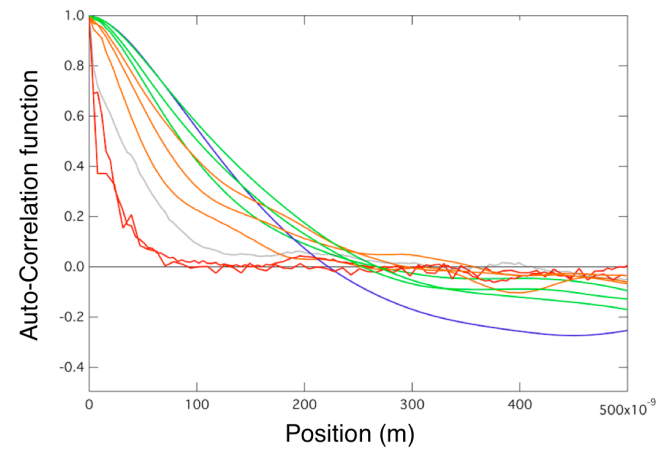

Figure 2. Root mean square roughness and autocorrelation functions from the AFM data. A) RMS roughness is plotted against the cumulative charge transferred (assuming 100\% efficiency). The dip in RMS roughness suggest island coalescence between the first and second pulse at $5 \mathrm{~mA} / \mathrm{cm} 2$. B) Auto-correlation function versus distance for the series of pulses.

USAXS data were obtained using the same pulse sequence described above. Figure 3 shows that the scattering intensity evolves during sequential pulses. There are two main effects. First a peak develops near $0.01 \mathrm{~A}^{-1}$, which increase in magnitude and shifts to lower wave vector (q) values with each pulse. We model this peak as a lognormal distribution of spherical particles. The second effect is a change in slope and magnitude in the linear region at low q between approximately 0.0001 and $0.001 \mathrm{~A}^{-1}$. We model this region as a power law fit.

(a)

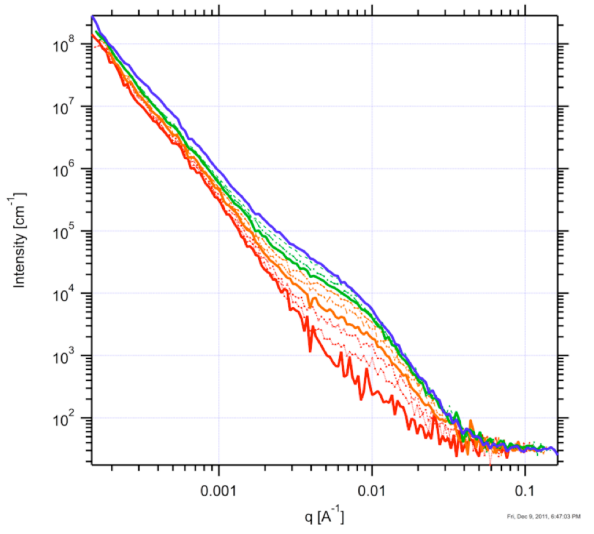

(b)

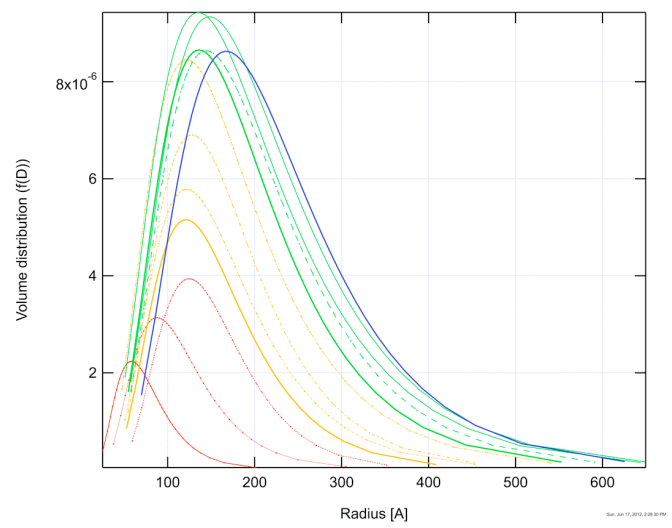

Figure 3. Ultra Small Angle Xray Scattering Data and Model Fits. a) Intensity versus scattering vector data for the sequence of voltage pulses described within the text. The solution background has been subtracted. b) Volume distribution versus radius for the model fits of the Guinier near $0.01 \mathrm{~A}^{-1}$. Fits assume a log-normal distribution of spherical particles. 
In the region near $0.01 \mathrm{~A}^{-1}$ the fits show the growth of nanoparticles with radii that begin at about $8 \mathrm{~nm}$ and grow to $12 \mathrm{~nm}$ during the first three pulses at $-1 \mathrm{~mA} / \mathrm{cm}^{2}$. The next three pulses at $-2 \mathrm{~mA} / \mathrm{cm}^{2}$ each increase the total scatter volume of the particles but do not increase the size appreciably. The $-5 \mathrm{~mA} / \mathrm{cm}^{2}$ pulses increase the radius to 13-15 $\mathrm{nm}$ and $-10 \mathrm{~mA} / \mathrm{cm}^{2}$ to $17 \mathrm{~nm}$.

It should be noted that the AFM data show features approximately an order of magnitude larger than those indicated by USAXS. At this point we do not fully understand the discrepancy between the AFM and USAXS results, however there are a couple possibilities that we are pursuing. The first is that is that electrodeposition within the geometry of the USAXS cell produces a different surface morphology than it does in the AFM cell due to differences in flow. In this instance we would expect the AFM results to be closer to diffusion-limited growth whereas the fast flow of the USAXS cell would be closer to reaction limited. We are testing this hypothesis by removing the sample from the USAXS cell after a series of pulses to examine the surface morphology ex situ using SEM and AFM.

An interesting possibility for the differences between the USAXS and AFM data is that the zinc growth occurs via particles, which aggregate into larger particles structures, much like one would observe with a snowflake. In this case, the particle distribution observed by USAXS (Figure 3b) would suggest that the islands observed by AFM are composed of smaller particles that have a 10-20 nm size range. The USAXS data samples the entire volume, which in this scenario would have voids in it that are not observed from the surface topography imaged by AFM. A cross-sectional TEM analysis should indicate whether this hypothesis is valid.

\section{Conclusions and Outlook}

Our first experiments demonstrate that we can successfully couple AFM and Xray scattering experiments in aggressive battery electrolytes over a range of current densities. Future experiments will examine temporal evolution at one current density, which will allow more quantitative modeling of the surface dynamics. This work validates the applicability of the techniques over a range of current densities.

\section{Acknowledgments}

We thank Jan Ilavsky for his superb help with modeling and interpretation of the USAXS data. We thank Troy Barbee for providing the very low surface roughness sputterdeposited copper substrates used in the AFM experiments. Portions of this work performed under the auspices of the U.S. Department of Energy by Lawrence Livermore National Laboratory under Contract DE-AC52-07NA27344. This project was supported by Lab directed Research and Development Grant 12-LW-030.

\section{References}

1. S. Torres and B. El-Dasher, Manuscript in preparation (2012).

2. J. Ilavsky and P. R. Jemian, J. Appl. Cryst., 42, 347 (2009). 